\title{
Quantum Theory of the Optical and Electronic Properties of Semiconductors
}

Fifth Edition 
This page intentionally left blank 


\section{Hartmut Haug}

Goethe-Universität Frankfurt, Germany

\section{Stephan W. Koch}

Philipps-Universität Marburg, Germany

\section{Quantum Theory of the Optical and Electronic Properties of Semiconductors}

Fifth Edition 
Published by

World Scientific Publishing Co. Pte. Ltd.

5 Toh Tuck Link, Singapore 596224

USA office: 27 Warren Street, Suite 401-402, Hackensack, NJ 07601

UK office: 57 Shelton Street, Covent Garden, London WC2H 9HE

\section{British Library Cataloguing-in-Publication Data}

A catalogue record for this book is available from the British Library.

\section{QUANTUM THEORY OF THE OPTICAL AND ELECTRONIC PROPERTIES OF SEMICONDUCTORS - Fifth Edition}

Copyright (C) 2009 by World Scientific Publishing Co. Pte. Ltd.

All rights reserved. This book, or parts thereof, may not be reproduced in any form or by any means, electronic or mechanical, including photocopying, recording or any information storage and retrieval system now known or to be invented, without written permission from the Publisher.

For photocopying of material in this volume, please pay a copying fee through the Copyright Clearance Center, Inc., 222 Rosewood Drive, Danvers, MA 01923, USA. In this case permission to photocopy is not required from the publisher.

ISBN-13 978-981-283-883-4

ISBN-10 981-283-883-X

ISBN-13 978-981-283-884-1 (pbk)

ISBN-10 981-283-884-8 (pbk)

Printed in Singapore. 


\section{Preface}

The electronic properties of semiconductors form the basis of the latest and current technological revolution, the development of ever smaller and more powerful computing devices, which affect not only the potential of modern science but practically all aspects of our daily life. This dramatic development is based on the ability to engineer the electronic properties of semiconductors and to miniaturize devices down to the limits set by quantum mechanics, thereby allowing a large scale integration of many devices on a single semiconductor chip.

Parallel to the development of electronic semiconductor devices, and no less spectacular, has been the technological use of the optical properties of semiconductors. The fluorescent screens of television tubes are based on the optical properties of semiconductor powders. The red light of GaAs light emitting diodes is known to all of us from the displays of domestic appliances, and semiconductor lasers are used to read optical discs and to write in laser printers. Furthermore, fiber-optic communications, whose light sources, amplifiers and detectors are again semiconductor electro-optical devices, are expanding the capacity of the communication networks dramatically.

Semiconductors are very sensitive to the addition of carriers, which can be introduced into the system by doping the crystal with atoms from another group in the periodic system, electronic injection, or optical excitation. The electronic properties of a semiconductor are primarily determined by transitions within one energy band, i.e., by intraband transitions, which describe the transport of carriers in real space. Optical properties, on the other hand, are connected with transitions between the valence and conduction bands, i.e., with interband transitions. However, a strict separation is impossible. Electronic devices such as a $p$ - $n$ diode can only be under- 
stood if one considers also interband transitions, and many optical devices cannot be understood if one does not take into account the effects of intraband scattering, carrier transport and diffusion. Hence, the optical and electronic semiconductor properties are intimately related and should be discussed jointly.

Modern crystal growth techniques make it possible to grow layers of semiconductor material which are narrow enough to confine the electron motion in one dimension. In such quantum-well structures, the electron wave functions are quantized like the standing waves of a particle in a square well potential. Since the electron motion perpendicular to the quantumwell layer is suppressed, the semiconductor is quasi-two-dimensional. In this sense, it is possible to talk about low-dimensional systems such as quantum wells, quantum wires, and quantum dots which are effectively two, one and zero dimensional.

These few examples suffice to illustrate the need for a modern textbook on the electronic and optical properties of semiconductors and semiconductor devices. There is a growing demand for solid-state physicists, electrical and optical engineers who understand enough of the basic microscopic theory of semiconductors to be able to use effectively the possibilities to engineer, design and optimize optical and electronic devices with certain desired characteristics.

Many results in the different chapters are developed in parallel first for bulk material, and then for quasi-two-dimensional quantum wells and for quasi-one-dimensional quantum wires, respectively. Semiconductor quantum dots are treated in a separate chapter. The semiconductor Bloch equations have a central position. They are formulated not only for free particles in various dimensions, but also in the Landau basis for low-dimensional electrons in strong magnetic fields or in the basis of quantum dot eigenfunctions. Correlation and scattering effects are introduced at different levels of approximation. Particularly, the relaxation and the dephasing in the Bloch equations are treated not only within the semi-classical Boltzmann kinetics, but also within quantum kinetics, which is needed for ultrafast semiconductor spectroscopy. The applications of these equations to timedependent and coherent phenomena in semiconductors are presented in the chapters dealing with the excitonic optical Stark effect and various nonlinear wave-mixing configurations. The discussion of the nonequilibrium Green's function theory contains both introductory material as well as applications to Coulomb carrier scattering and time-dependent screening. The final chapter in this book deals with quantum optical effects in semicon- 
ductors. A cluster-expansion scheme is used to treat the Coulombic and quantum optical correlations consistently. The semiconductor luminescence equations are derived and solved for several examples.

This book is written for graduate-level students or researchers with general background in quantum mechanics as an introduction to the quantum theory of semiconductors. The necessary many-particle techniques, such as field quantization and Green's functions are developed explicitly. Wherever possible, we emphasize the motivation of a certain derivation and the physical meaning of the results, avoiding the discussion of formal mathematical aspects of the theory. The book, or parts of it, can serve as textbook for use in solid state physics courses, or for more specialized courses on electronic and optical properties of semiconductors and semiconductor devices. Especially the later chapters establish a direct link to current research in semiconductor physics.

Frankfurt and Marburg

October 2008

Hartmut Haug Stephan W. Koch 


\section{About the authors}

Hartmut Haug obtained his Ph.D (Dr. rer. nat., 1966) in Physics at the University of Stuttgart. From 1967 to 1969, he was a faculty member at the Department of Electrical Engineering, University of Wisconsin in Madison. After working as a member of the scientific staff at the Philips Research Laboratories in Eindhoven from 1969 to 1973, he joined the Institute of Theoretical Physics of the University of Frankfurt, where he was a full professor from 1975 to 2001 and currently is an emeritus. He has been a visiting scientist at many international research centers and universities.

Stephan W. Koch obtained his Ph. D. (Dr. phil. nat., 1979) in Physics at the University of Frankfurt. Until 1993 he was a full professor both at the Department of Physics and at the Optical Sciences Center of the University of Arizona, Tucson (USA). In the fall of 1993, he joined the Philipps-University of Marburg where he is a full professor of Theoretical Physics. He is a Fellow of the Optical Society of America. He received the Leibniz prize of the Deutsche Physikalische Gesellschaft (1997) and the Max-Planck Research Prize of the Humboldt Foundation and the MaxPlanck Society (1999). 


\section{Contents}

Preface $\quad$ v

1. Oscillator Model 1

1.1 Optical Susceptibility . . . . . . . . . . . . . . 2

1.2 Absorption and Refraction . . . . . . . . . . . . . 6

1.3 Retarded Green's Function . . . . . . . . . . . . . . . 12

2. Atoms in a Classical Light Field 17

2.1 Atomic Optical Susceptibility . . . . . . . . . . . . . . 17

2.2 Oscillator Strength . . . . . . . . . . . . . . . . . . 21

2.3 Optical Stark Shift . . . . . . . . . . . . . . . . 23

3. Periodic Lattice of Atoms 29

3.1 Reciprocal Lattice, Bloch Theorem . . . . . . . . . . . 29

3.2 Tight-Binding Approximation . . . . . . . . . . . . . . 36

$3.3 \mathrm{k} \cdot \mathrm{p}$ Theory . . . . . . . . . . . . . . . . . . . . . . 41

3.4 Degenerate Valence Bands . . . . . . . . . . . . . . 45

4. Mesoscopic Semiconductor Structures 53

4.1 Envelope Function Approximation . . . . . . . . . . . 54

4.2 Conduction Band Electrons in Quantum Wells . . . . . . 56

4.3 Degenerate Hole Bands in Quantum Wells . . . . . . . . . 60

5. Free Carrier Transitions 65

5.1 Optical Dipole Transitions . . . . . . . . . . . . . . 65

5.2 Kinetics of Optical Interband Transitions _ . . . . . . . 69 
5.2.1 Quasi- $D$-Dimensional Semiconductors . . . . . . 70

5.2.2 Quantum Confined Semiconductors with Subband Structure . . . . . . . . . . . . 72

5.3 Coherent Regime: Optical Bloch Equations . . . . . . . . 74

5.4 Quasi-Equilibrium Regime:

Free Carrier Absorption . . . . . . . . . . . . . . . 78

6. Ideal Quantum Gases $\quad 89$

6.1 Ideal Fermi Gas . . . . . . . . . . . . . . . . . . . . . . . . 90

6.1.1 Ideal Fermi Gas in Three Dimensions . . . . . . . . . 93

6.1.2 Ideal Fermi Gas in Two Dimensions . . . . . . . . . . 97

6.2 Ideal Bose Gas . . . . . . . . . . . . . . . . . . . 97

6.2.1 Ideal Bose Gas in Three Dimensions . . . . . . . . . 99

6.2.2 Ideal Bose Gas in Two Dimensions . . . . . . . . . . 101

6.3 Ideal Quantum Gases in D Dimensions . . . . . . . . . . . . 101

7. Interacting Electron Gas 107

7.1 The Electron Gas Hamiltonian _. . . . . . . . . . 107

7.2 Three-Dimensional Electron Gas . . . . . . . . . . . . 113

7.3 Two-Dimensional Electron Gas . . . . . . . . . . . . . . . 119

7.4 Multi-Subband Quantum Wells . . . . . . . . . . . . . 122

7.5 Quasi-One-Dimensional Electron Gas . . . . . . . . . . . 123

8. Plasmons and Plasma Screening 129

8.1 Plasmons and Pair Excitations _. . . . . . . . . . 129

8.2 Plasma Screening . . . . . . . . . . . . . . . . . 137

8.3 Analysis of the Lindhard Formula . . . . . . . . . . . 140

8.3.1 Three Dimensions . . . . . . . . . . . . . . . . 140

8.3 .2 Two Dimensions . . . . . . . . . . . . . . . . . . . 143

8.3 .3 One Dimension . . . . . . . . . . . . . . . . 145

8.4 Plasmon-Pole Approximation . . . . . . . . . . . . . 146

9. Retarded Green's Function for Electrons 149

9.1 Definitions . . . . . . . . . . . . . . . . . . . . 149

9.2 Interacting Electron Gas . . . . . . . . . . . . . 152

9.3 Screened Hartree-Fock Approximation . . . . . . . . . . . 156 
10. Excitons 163

10.1 The Interband Polarization . . . . . . . . . . . . . . 164

10.2 Wannier Equation . . . . . . . . . . . . . . . . 169

10.3 Excitons . . . . . . . . . . . . . . . . . . . . 173

10.3.1 Three- and Two-Dimensional Cases . . . . . . . . . 174

10.3.2 Quasi-One-Dimensional Case . . . . . . . . . . 179

10.4 The Ionization Continuum . . . . . . . . . . . . . . . 181

10.4.1 Three- and Two-Dimensional Cases . . . . . . . . . . 181

10.4.2 Quasi-One-Dimensional Case . . . . . . . . . . 183

10.5 Optical Spectra . . . . . . . . . . . . . . . . . 184

10.5.1 Three- and Two-Dimensional Cases . . . . . . . 186

10.5.2 Quasi-One-Dimensional Case . . . . . . . . . . 189

11. Polaritons 193

11.1 Dielectric Theory of Polaritons . . . . . . . . . . . . . 193

11.1.1 Polaritons without Spatial Dispersion and Damping . . . . . . . . . . . . 195

11.1.2 Polaritons with Spatial Dispersion and Damping . . 197

11.2 Hamiltonian Theory of Polaritons . . . . . . . . . . . . . . . 199

11.3 Microcavity Polaritons . . . . . . . . . . . . . . 206

12. Semiconductor Bloch Equations 211

12.1 Hamiltonian Equations . . . . . . . . . . . . . . . . . 211

12.2 Multi-Subband Microstructures . . . . . . . . . . . . . . 219

12.3 Scattering Terms . . . . . . . . . . . . . . . 221

12.3.1 Intraband Relaxation . . . . . . . . . . . . . . 226

12.3.2 Dephasing of the Interband Polarization . . . . . . . 230

12.3.3 Full Mean-Field Evolution of the Phonon-Assisted Density Matrices . . . . . . . . . . . . . 231

13. Excitonic Optical Stark Effect 235

13.1 Quasi-Stationary Results . . . . . . . . . . . . . . . . 237

13.2 Dynamic Results . . . . . . . . . . . . . . . . 246

13.3 Correlation Effects . . . . . . . . . . . . . . . 255

14. Wave-Mixing Spectroscopy 269

14.1 Thin Samples . . . . . . . . . . . . . . . . . . . . . . . . . . . . 271

14.2 Semiconductor Photon Echo . . . . . . . . . . . . . 275 
15. Optical Properties of a Quasi-Equilibrium Electron-Hole Plasma

15.1 Numerical Matrix Inversion . . . . . . . . . . . . . 287

15.2 High-Density Approximations . . . . . . . . . . . . . . 293

15.3 Effective Pair-Equation Approximation . . . . . . . . . . 296

15.3.1 Bound states . . . . . . . . . . . . . . . 299

15.3.2 Continuum states . . . . . . . . . . . . . 300

15.3.3 Optical spectra . . . . . . . . . . . . . . . 300

16. Optical Bistability 305

16.1 The Light Field Equation . . . . . . . . . . . . . . . . 306

16.2 The Carrier Equation . . . . . . . . . . . . . . . 309

16.3 Bistability in Semiconductor Resonators . . . . . . . . . 311

16.4 Intrinsic Optical Bistability . . . . . . . . . . . . . 316

17. Semiconductor Laser 321

17.1 Material Equations . . . . . . . . . . . . . . . . . 322

17.2 Field Equations . . . . . . . . . . . . . . . . . 324

17.3 Quantum Mechanical Langevin Equations . . . . . . . . . 328

17.4 Stochastic Laser Theory . . . . . . . . . . . . . . . . 335

17.5 Nonlinear Dynamics with Delayed Feedback . . . . . . . . . 340

18. Electroabsorption 349

18.1 Bulk Semiconductors . . . . . . . . . . . . . . . . 349

18.2 Quantum Wells . . . . . . . . . . . . . . . . 355

18.3 Exciton Electroabsorption . . . . . . . . . . . . . . 360

18.3.1 Bulk Semiconductors . . . . . . . . . . . . 360

18.3.2 Quantum Wells . . . . . . . . . . . . . 368

19. Magneto-Optics 371

19.1 Single Electron in a Magnetic Field . . . . . . . . . . . . . 372

19.2 Bloch Equations for a Magneto-Plasma . . . . . . . . . . . . 375

19.3 Magneto-Luminescence of Quantum Wires . . . . . . . 378

20. Quantum Dots 383

20.1 Effective Mass Approximation . . . . . . . . . . . . . . . . . . 383

20.2 Single Particle Properties . . . . . . . . . . . . . . . . . . . . 386

20.3 Pair States . . . . . . . . . . . . . . . . . . . 388 
20.4 Dipole Transitions . . . . . . . . . . . . . . . . . . 392

20.5 Bloch Equations . . . . . . . . . . . . . . . 395

20.6 Optical Spectra . . . . . . . . . . . . . . . 396

21. Coulomb Quantum Kinetics 401

21.1 General Formulation . . . . . . . . . . . . . . . . . . . . . 402

21.2 Second Born Approximation . . . . . . . . . . . . . . 408

21.3 Build-Up of Screening . . . . . . . . . . . . . . . . 413

22. Quantum Optical Effects 421

22.1 Quantum Optics for Semiconductors . . . . . . . . . . 421

22.2 Cluster Expansion . . . . . . . . . . . . . . . . . . 424

22.2.1 Cluster Expansion for Fermions . . . . . . . . . . . . 424

22.2.2 Quantum Optical Cluster Expansion . . . . . . . . . 428

22.3 Semiconductor Luminescence Equations . . . . . . . . . . 429

22.4 Quasi-Stationary Luminescence . . . . . . . . . . . . . . 432

$\begin{array}{lll}\text { Appendix A } & \text { Field Quantization } & 437\end{array}$

A.1 Lagrange Functional . . . . . . . . . . . . . . . . . 437

A.2 Canonical Momentum and Hamilton Function . . . . . . . . 442

A.3 Quantization of the Fields . . . . . . . . . . . . . . . 444

Appendix B Contour-Ordered Green's Functions 451

B.1 Interaction Representation . . . . . . . . . . . . . . 452

B.2 Langreth Theorem . . . . . . . . . . . . . . . . . 455

B.3 Equilibrium Electron-Phonon Self-Energy . . . . . . . . . 458

$\begin{array}{ll}\text { Index } & 461\end{array}$ 